\title{
É normal dar à luz em casa: A busca pela revalorização do ambiente domiciliar como espaço adequado para o momento do parto
}

It's normal giving birth at home: The search for the revaluation of the home environment as an adequate space for the moment of childbirth

Es normal dar a luz en casa: La búsqueda de la revalorización del entorno del hogar como espacio adecuado para el momento del parto

Rúbia Laniêdja Oliveira Silva ORCID: https://orcid.org/0000-0002-3963-2182 Faculdade de Tecnologia e Ciências, Brasil E-mail: rubia.lc@hotmail.com

Ilana Maria Brasil do Espírito Santo ORCID: https://orcid.org/0000-0001-9591-2704 Centro Universitário Internacional UNINTER, Brasil E-mail: ilaleao@outlook.com

Reginaldo Santana Lima ORCID: https://orcid.org/0000-0002-7924-6833

Faculdade de Tecnologia e Ciências, Brasil E-mail: naldosanlima@hotmail.com

Hallyson Leno Lucas da Silva ORCID: https://orcid.org/0000-0001-7237-1706 Faculdades Integradas de Patos, Brasil E-mail: hallysonlenolucas@hotmail.com

Michelinne Shirley Pinheiro dos Santos ORCID: https://orcid.org/0000-0002-1334-5345

Universidade Estadual do Piauí, Brasil

E-mail: michelinnesantos@yahoo.com.br

Maria de Lourdes Lima Oliveira Cardoso ORCID: https://orcid.org/0000-0002-1515-3793 Centro Universitário Santo Agostinho, Brasil E-mail: luma-82@outlook.com

Maria da Guia Damasceno de Ananias ORCID: https://orcid.org/0000-0001-5266-3197 Faculdade Evangélica do Meio Norte, Brasil E-mail: mariadaguiada@ hotmail.com

Aline Stefhane Coutinho Coelho ORCID: https://orcid.org/0000-0003-0933-4659 Centro Universitário UNINOVAFAPI, Brasil

E-mail: allynnesthephany@hotmail.com Aline Sousa da Luz

ORCID: https://orcid.org/0000-0002-5022-5350 Universidade Cândido Mendes, Brasil

E-mail: alinneluzz17@gmail.com

Elizângela Vieira de Araújo ORCID: https://orcid.org/0000-0002-5895-4021 Centro Universitário Santo Agostinho, Brasil E-mail: elizangela.vieira80@gmail.com Izabel Pereira Duarte

ORCID: https://orcid.org/0000-0002-7000-482X Unidades Integradas de Pós-Graduação, Brasil E-mail: dizabel615@gmail.com

Emília Vieira de Holanda Lira ORCID: https://orcid.org/0000-0003-1466-4433

Universidade Federal do Maranhão, Brasil E-mail: emilialira30@hotmail.com

Juliana de Jesus Nogueira dos Santos ORCID: https://orcid.org/0000-0002-1245-8524

Centro Universitário Santo Agostinho, Brasil E-mail: Jiuliana_nogueira@hotmail.com 
Francisco Lucas de Lima Fontes ORCID: https://orcid.org/0000-0003-1880-9329 Universidade Federal do Piaú, Brasil E-mail: lucasfontesenf@ufpi.edu.br

\begin{abstract}
Resumo
Objetivou-se com o presente estudo evidenciar a revalorização do ambiente domiciliar como espaço adequado para o momento do parto. Trata-se de uma revisão de literatura do tipo bibliográfica/narrativa, fundamentada na dimensão exploratória. As fontes para busca dos referenciais envolveram a base de dados Portal de Periódicos da Coordenação de Aperfeiçoamento de Pessoal de Nível Superior (CAPES), a biblioteca virtual Scientific Electronic Library Online (SciELO) e o buscador eletrônico Google Scholar. O domicílio enquanto local de cuidado no parto traz vários elementos que favorecem um dos principais requisitos para o resgate da humanização no processo de nascimento: a autonomia da mulher. No domicílio ela se torna sujeito ativo de seu parto, resgatando para si o próprio parto e o controle sobre seu corpo, tendo a oportunidade de atuar, de fazer suas escolhas com segurança, sem se inibir. No domicílio a mulher se sente segura, rodeada de pessoas que a amam, podendo expressar seus sentimentos e ser autêntica nos seus comportamentos e condutas. Com esta revisão verifica-se que o domicílio pode ser singular e importante espaço para o cuidado, pois a partir do conhecimento das características específicas deste ambiente e de seus membros é possível que os profissionais que assistem a gestante/parturiente desenvolvam ações de cuidado mais contextualizadas e efetivas.
\end{abstract}

Palavras-chave: Parto domiciliar; Humanização do parto; Assistência ao parto.

\begin{abstract}
The objective of this study was to highlight the revaluation of the home environment as an adequate space for the moment of birth. This is a literature review of the bibliographic/narrative type, based on the exploratory dimension. The sources for searching the referentials involved the database of the dados Portal de Periódicos da Coordenação de Aperfeiçoamento de Pessoal de Nível Superior (CAPES), the virtual library Scientific Electronic Library Online (SciELO) and the electronic search engine Google Scholar. The home as a place of care in childbirth brings several elements that favor one of the main requirements for the rescue of humanization in the birth process: the woman's autonomy. At home, she becomes an active subject of her birth, rescuing her own birth and control over her body, having the opportunity to act, to make her choices safely, without being inhibited. At home, the woman feels safe, surrounded by people who love her, being able to express her feelings and be authentic in her behaviors and behaviors. With this review, it appears that the home can be unique and an important space for care, because from the knowledge of the specific characteristics of this environment and its members, it is possible that the professionals who assist the pregnant woman/parturient to develop more contextualized care actions and effective.
\end{abstract}

Keywords: Home childbirth; Humanizing childbirth; Midwifery.

\title{
Resumen
}

El objetivo de este estudio fue resaltar la revalorización del ambiente domiciliario como un espacio adecuado para el momento del parto. Se trata de una revisión de la literatura de tipo bibliográfica/narrativa, basada en la dimensión exploratoria. Las fuentes para la búsqueda de los referenciales involucraron la base de datos del Portal de Periódicos da Coordenação de Aperfeiçoamento de Pessoal de Nível Superior (CAPES), la biblioteca virtual Scientific Electronic Library Online (SciELO) y el buscador electrónico Google Scholar. El hogar como lugar de cuidado en el parto trae varios elementos que favorecen uno de los principales requisitos para el rescate de la humanización en el proceso del parto: la autonomía de la mujer. En casa, se convierte en un sujeto activo de su nacimiento, rescatando su propio nacimiento y el control de su cuerpo, teniendo la oportunidad de actuar, de tomar sus decisiones de forma segura, sin inhibirse. En casa, la mujer se siente segura, rodeada de personas que la quieren, pudiendo expresar sus sentimientos y ser auténtica en sus comportamientos y comportamientos. Con esta revisión, se desprende que el hogar puede ser único y un espacio importante para el cuidado, pues a partir del conocimiento de las características específicas de este entorno y sus integrantes, es posible que los profesionales que asisten a la gestante/parturienta se desarrollen más Acciones asistenciales contextualizadas y efectivas.

Palabras clave: Parto domiciliario; Parto humanizado; Partería.

\section{Introdução}

O parto é uma etapa na vida da mulher, um processo natural na história da população humana. A gestação e o parto constituem uma das experiências humanas mais significativas e impactantes para o corpo feminino, que podem ter resultados positivos ou negativos e influenciar experiências futuras (Mattos, Vandenberghe \& Martins, 2016). Segundo Barros et al. (2011) durante a trajetória de vida, a mulher passa por diversos processos que favorecem mudanças em seu comportamento e, 
nesse sentido, o parto constitui um evento de grandes transformações para a parturiente, devendo ser dada a ela autonomia e capacidade de decisões sobre o seu corpo no momento de parir seu filho.

Até o século XVIII, o parto era considerado um ritual de mulheres e não um ato médico, já que ficava a cargo das parteiras. No final do século XIX, os médicos obstetras passaram a empreender campanhas para transformar o parto em um evento controlado, o que se efetivou na metade do século XX. O cenário do parto domiciliar alterou-se foi extinto paulatinamente, uma vez que a medicina transformou esse evento, que é fisiológico, em um evento patológico, que necessita na maioria das vezes de tratamento medicamentoso e cirúrgico, com predominância da assistência hospitalar ao parto, tornando-o, a partir daí, um ato institucionalizado (Denipote et al., 2020; Medeiros et al., 2020). Para Dias e Deslandes (2006) a medicalização do processo negou a capacidade autônoma parturitiva da mulher e definiu para ela um papel passivo na gestação e no nascimento, o que ocasionou a necessidade de hospitalização e de procedimentos médicos.

O Brasil vivencia hoje uma epidemia de operações cesarianas, com taxas superiores às recomendadas pela Organização Mundial de Saúde (2015), que seria entre 10 e 15\%. De acordo com a Comissão Nacional de Incorporação de Tecnologias no Sistema Único de Saúde (2015), em 2015 as cesáreas atingiram a marca de 56\%, sendo 40\% no setor público. No setor privado a situação é ainda mais alarmante, com taxas de 88\% em 2012 e 85\% em 2015, que correspondem a duas vezes mais que o setor público e duas vezes mais que a média nacional.

Referindo-se ao número excessivo de cesáreas realizadas no Brasil, Rattner (2009) afirma que este passou a ser o método "normal" de parir, contribuindo para o aumento da morbimortalidade materna e perinatal, bem como para a elevação dos gastos desnecessários de recursos financeiros e humanos. Constata-se que se constituiu um processo de desnaturalização do parto onde se subentende, no discurso biomédico, a incapacidade da mulher de parir.

Diante desta realidade, o Ministério da Saúde do Brasil, em consenso com a tendência dos principais organismos internacionais da área da saúde e dos movimentos de humanização, elegeu como tática a implantação da Política Nacional de Humanização (PNH) que, no contexto do parto e nascimento, tem conformidade com o Programa de Humanização do Prénatal e Nascimento e com a Política Nacional de Atenção Integral à Saúde da Mulher. O Programa se respalda na premissa de retomar o parto enquanto evento fisiológico e devolver o protagonismo da mulher, buscando promover um nascimento mais humano, digno e respeitoso (Brasil, 2017). Nesse movimento de humanização, com base nas evidências científicas houve a necessidade de modificar as rotinas, as condutas e a estrutura física dos hospitais, além da sensibilização dos profissionais com vistas à oferta de uma assistência humanizada com redução de intervenções desnecessárias.

Há 11 anos, Nascimento et al. (2010) já relatavam em seu estudo as grandes mudanças no cenário da assistência obstétrica, com retomada de valores que vão além dos aspectos científicos e tecnológicos, apontando para o resgate do modelo histórico do nascimento, trazendo novamente o ambiente domiciliar como local propício para o parto. Neste contexto, enfermeiros obstetras despontam na ascensão ao parto domiciliar planejado, no intento de retomar a qualidade da assistência à parturição para a parturiente e para o recém-nascido.

Atualmente, a assistência ao parto apresenta esse movimento de mudanças para as práticas culturais menos intervencionistas. Observa-se a busca por parte da gestante pelo parto em casa, conhecido por parto domiciliar planejado, em grandes capitais do Brasil. A residência apresenta-se para essas parturientes como um ambiente seguro e viável para darem à luz (Cursino \& Benincasa, 2020; Santos et al., 2020; Mocheuti et al., 2020).

Diante do ressurgimento deste novo quadro assistencial, Mattos, Vandenberghe e Martins (2016) enfatizam em sua pesquisa que o parto domiciliar planejado refere-se aos nascimentos que acontecem em domicilio, de forma intencional e programada pela mãe, juntamente com o profissional responsável pela assistência a ser prestada que a acompanhou previamente no pré-natal, prevenindo, assim, os riscos associados ao seu parto. Esta modalidade de nascimento permite maior controle do ambiente, por parte da parturiente e familiares, envolvidos no evento do parto. 
O interesse de estudo pela temática surgiu mediante vivências durante trabalhos executados em centros obstétricos, com a identificação de lacunas entre o que foi visto na teoria e o que é colocado em prática nesses ambientes. Constatou-se que na assistência diária não havia, por parte dos profissionais, a observância do documento criado pela Organização Mundial da Saúde que versa sobre as "boas práticas de atenção ao parto e ao nascimento", que recomenda que o parto vaginal de baixo risco deve ser realizado com o mínimo possível de intervenções para evitar uma vivência traumática tanto para a mãe quanto para o bebê. Diante dessa perspectiva, o parto domiciliar surge como uma opção diferenciada, em que a mulher participa ativamente de todas as decisões referentes ao seu parto e permite uma relação de confiança e afetividade entre o profissional que a assiste e a família envolvida. Ancorando-se no que foi brevemente explanado, o objetivo do presente estudo foi evidenciar a revalorização do ambiente domiciliar como espaço adequado para o momento do parto.

\section{Metodologia}

Trata-se de uma revisão de literatura do tipo bibliográfica/narrativa, fundamentada na dimensão exploratória. A pesquisa bibliográfica abrange toda bibliografia tornada pública em relação ao tema de estudo, onde são consideradas para construção do estudo publicações avulsas, boletins, jornais, revistas, livros, pesquisas, monografias, teses, material cartográfico, entre outros. São considerados até meios de comunicação orais como rádio, gravação em fita magnética e audiovisuais como filmes e televisão. Sua função e colocar o pesquisador em contato direto com o que foi escrito, dito ou filmado (Marconi \& Lakatos, 2017).

As fontes para busca dos referenciais envolveram a base de dados Portal de Periódicos da Coordenação de Aperfeiçoamento de Pessoal de Nível Superior (CAPES), a biblioteca virtual Scientific Electronic Library Online (SciELO) e o buscador eletrônico Google Scholar.

A busca por materiais em mais de uma fonte ocorreu para ampliar o leque de acessos à construção da revisão bibliográfica/narrativa. Na procura pelos referenciais adotaram-se os seguintes Descritores em Ciências da Saúde (DeCS): "parto domiciliar", "humanização do parto" e "assistência ao parto". Incluíram-se referenciais (artigos, monográficas, dissertações, teses e outros produtos técnicos sobre o tema estudado) disponíveis na íntegra e nas línguas portuguesa, inglesa ou espanhola. Decidiu-se não aplicar recorte temporal na busca dos referenciais.

Os referenciais foram escolhidos buscando-se produções que atendessem ao objetivo determinado pelo estudo e tema explorado. Assim, os entendimentos que se realizaram dos referenciais ficaram sujeitos ao viés de seleção, com interpretações e análise crítica subjetivos a cargo dos autores da revisão.

\section{Resultados e Discussão}

O parto é um evento cultural que teve início com a existência da mulher na humanidade. Embora o corpo feminino seja preparado fisiologicamente durante a gestação para a expulsão do concepto, ao longo da história o parto sofreu implicações sociais e culturais (Possati et al., 2017; Silva et al., 2019).

O modelo de assistência ao nascimento, culturalmente teve início com a presença da parteira, cujo conhecimento era exclusivamente prático, adquirido mediante experiências dos seus próprios partos, por acompanharem as parteiras ou por acompanharem suas próprias mães, passando a arte de partejar por diversas gerações. Os partos eram sempre realizados em domicílio, permitindo a presença e participação da família durante todo processo, mas ao longo dos anos a prática obstétrica passou por grandes mudanças no processo de assistir ao parto (Fabrizzio et al., 2019; Lima et al., 2021).

As parteiras possuem uma forma particular de saber e fazer. Na sua atuação fazem uso de conhecimentos tradicionais, baseados em experiências repassadas oralmente de mãe para filha. Os conhecimentos são advindos de crenças, tradições, misticismo, fruto de uma prática empírica e repetida. O saber da parteira não está relacionado à educação formal, mas às 
experiências acumuladas ao longo dos anos (Cardoso \& Nascimento, 2019; Kappaun \& Costa, 2020).

O Ministério da Saúde, a partir de 2000, adotou várias iniciativas para melhorar a atenção à gestação, ao parto, ao nascimento e ao puerpério. Dentre elas, encontrava-se o Programa Trabalhando com Parteiras Tradicionais, que reconduziu a melhorias do parto e do nascimento domiciliar assistidos por parteiras tradicionais na pauta de discussão com gestores estaduais e municipais, como uma responsabilidade do Sistema Único de Saúde e uma atribuição da Atenção Básica. Este programa visava resgatar e valorizar os saberes tradicionais, articulando-os com os científicos, ao considerar a riqueza cultural e da biodiversidade como elementos importantes para a produção de saúde, além de novos conhecimentos e de tecnologias (Brasil, 2010).

Davim e Menezes (2001) e Kruno e Bonilha (2004) relatam em seus estudos que, diferentemente das parteiras tradicionais, a atuação do enfermeiro obstetra teve seu início voltado para a atenção ao parto hospitalar, visando cumprir os propósitos do Ministério da Saúde sob outra abordagem, mas com atenção à mulher e à criança. Neste sentido, o enfermeiro obstetra apresentou-se como um profissional relevante na redução da mortalidade materno-infantil, bem como na prestação de uma assistência ao parto de qualidade.

A tecnologia e os estudos científicos proporcionaram avanços na melhoria da qualidade da assistência obstétrica. Entre esses avanços encontra-se a operação cesariana, procedimento anteriormente realizado apenas em mulheres mortas com o objetivo de salvar a vida da criança. Observa-se, contudo, que esse método passou a ser usado sem maiores justificativas, o que provoca uma medicalização excessiva em um ato que deveria ser natural, fisiológico, pertinente a qualquer mulher (Silva et al., 2020).

Estudos recentes (Denipote et al., 2020; Lessa et al., 2018) mostram que, assim como a parteira tradicional em regiões rurais, o enfermeiro obstetra vem crescendo como profissional autônomo no atendimento a partos domiciliares em regiões urbanas. Este profissional tem associado o seu trabalho ao modelo das parteiras tradicionais. A assistência mais efetiva seria, portanto, centrada nas necessidades das parturientes, em vez de organizada segundo as necessidades de instituições e de profissionais.

Para Mattos, Vandenberghe e Martins (2016) os preconceitos culturais sobre o parto ideal foram formados ao longo dos anos, no imaginário das pessoas. Construiu-se uma ideia de que o parto "bom" seria aquele sem dor, no ambiente hospitalar, com a garantia das tecnologias modernas. Neste sentido, a sociedade e os profissionais de saúde constroem pontos de vista acerca da assistência ao parto. Esta visão do parto como um procedimento cirúrgico de alta complexidade não deixa espaço para o parto domiciliar planejado.

A humanização da assistência demanda do profissional enfermeiro uma visão empática e holística, no sentido de compreender o outro. A humanização foi imposta como política pública de saúde, iniciando pelo ambiente hospitalar e, posteriormente, dirigida a todos os níveis de atenção à saúde. A política ganhou força social pela humanização do parto e nascimento, que influenciou a transformação da prática de muitos enfermeiros na assistência ao parto (Limeira et al., 2018).

Frank e Pelloso (2013) afirmam em seu estudo que, na visão dos profissionais, o domicílio enquanto local de cuidado no parto traz vários elementos que favorecem um dos principais requisitos para o resgate da humanização no processo de nascimento: a autonomia da mulher. No domicílio ela se torna sujeito ativo de seu parto, resgatando para si o próprio parto e o controle sobre seu corpo, tendo a oportunidade de atuar, de fazer suas escolhas com segurança, sem se inibir. No domicílio a mulher se sente segura, rodeada de pessoas que a amam, podendo expressar seus sentimentos e ser autêntica nos seus comportamentos e condutas.

Segundo Mattos, Vandenberghe e Martins (2016) em alguns países a escolha pelo parto domiciliar planejado está associado às razões econômicas, uma vez que o sistema de saúde destes países é de difícil acesso à população, sendo o domicílio escolhido por algumas famílias devido ao baixo custo. A confiança junto ao profissional, a tradição do trabalho de 
algumas enfermeiras e a preservação da cultura do parto domiciliar acabam conquistando maior espaço, comparado ao parto institucionalizado.

Embora em alguns países como Holanda, Canadá e Austrália o parto domiciliar represente um evento não somente reconhecido, como também estimulado pelo sistema público de saúde, no Brasil percebemos um cenário completamente diferente. O parto domiciliar, em nossa realidade nacional, ainda é visto com muito preconceito por grande parte de nossa sociedade, principalmente devido à divulgação de conceitos equivocados sobre o tema (Sanfelice et al., 2014).

Ainda nesse contexto, Sanfelice et al. (2014) acrescentam que, nestes países, o parto domiciliar é considerado uma modalidade de assistência tão segura quanto o parto hospitalar, evidenciada como uma experiência mais satisfatória às mulheres e suas famílias e, acima de tudo, representa um serviço potencialmente menos oneroso ao Estado. Essas questões compõem as principais justificativas para que o governo apoie e incentive a realização do parto domiciliar nos países de primeiro mundo.

Ao contrário do que ocorre nos países de primeiro mundo, no Brasil, as mulheres que optam pelo parto domiciliar são socialmente reconhecidas como irresponsáveis, desinformadas e adeptas de um modismo. Da mesma forma, os profissionais que optam em oferecer esse atendimento acabam sendo explicitamente coagidos pelos conselhos de classe, perseguidos e desmoralizados (Denipote et al., 2020; Santos et al., 2019).

O resgate do parto domiciliar e sua ascensão na atualidade remete à existência de uma oposição à condição e à concepção dominante na sociedade. Este modelo de atenção ao nascimento reconhece uma maior autonomia de profissionais que assistem a parturiente, como o enfermeiro obstetra. Isto não está ligado ao fato de desvincular-se do médico obstetra, e sim às conquistas do espaço de atuação de outras categorias profissionais (Frank \& Pelloso, 2013; Oliveira et al., 2021).

$\mathrm{Na}$ assistência domiciliar o profissional precisa ir além do conhecimento científico e prestar um cuidado totalmente humanizado marcado pelo afeto, pelo calor humano, companheirismo, inspirando confiança e segurança e preocupando-se com o bem-estar e conforto da mulher, isto contribui para um a evolução positiva do parto em uma atmosfera harmoniosa (Brasil, 2010).

\section{Considerações Finais}

A Organização Mundial da Saúde reconhece que o melhor lugar para o parto é aquele em que a mulher se sinta segura. Este ambiente pode ser o domicílio, o centro de parto normal ou o hospital-maternidade. A residência é um ambiente seguro para o nascimento, desde que seja uma decisão da mulher e família.

Após extensa leitura dos materiais de estudo, constata-se que o atendimento ao parto domiciliar é um modelo ainda pouco conhecido pela sociedade brasileira, o que contribui para a construção de uma percepção preconceituosa e errada a seu respeito, fato que dificulta a sua disseminação.

Pôde-se entender que o cenário que envolve o parto domiciliar planejado está sendo ampliado muito vagarosamente no Brasil. Sabe-se que, anteriormente, este modelo de atenção ao parto era mais centrado nas práticas culturais de comunidades isoladas, por parteiras tradicionais em algumas regiões carentes do país ou zonas rurais. Porém, o modelo atual tem como território os grandes centros urbanos, onde predomina a inserção de profissionais como o enfermeiro obstetra.

Com esta revisão verifica-se que o domić́lio pode ser singular e importante espaço para o cuidado, pois a partir do conhecimento das características específicas deste ambiente e de seus membros é possível que os profissionais que assistem a gestante/parturiente desenvolvam ações de cuidado mais contextualizadas e efetivas.

É relevante pontuar que existem informações e evidências científicas que fundamentam a proposta de assistência ao parto qualificada à mulher no processo parturitivo em domicílio. Também, reforça-se a posição de que são necessárias e urgentes a criação de políticas de saúde que permitam a inserção do parto domiciliar nos sistemas de saúde, público e privado, 
e ainda a criação de resoluções que amparem o resgate do domicílio como cenário de nascimento, como direito à cidadania da mulher brasileira.

\section{Referências}

Barros, W. L. L., Costa, E., Boeckmann, L. M. M., Reis, P. E. D., Leon, C. G. R. M. P., \& Funghetto, S. S. (2011). Humanizing delivery: a reality in a birth center? Revista de Enfermagem da UFPE, 5(1), 67-74.

Brasil (2015). Ministério da Saúde. Comissão Nacional de Incorporação de Tecnologias no Sistema Único de Saúde. Diretrizes de atenção à gestante: a operação cesariana. Brasília: Ministério da Saúde.

Brasil (2010). Ministério da Saúde. Secretaria de Atenção à Saúde. Parto e nascimento domiciliar assistidos por parteiras tradicionais: o Programa Trabalhando com Parteiras Tradicionais e experiências exemplares. Brasília: Ministério da Saúde.

Brasil (2017). Ministério da Saúde. Secretaria de Ciência, Tecnologia e Insumos Estratégicos. Diretrizes nacionais de assistência ao parto normal. Brasília: Ministério da Saúde.

Cardoso, M. A. S., \& Nascimento, R. (2019). O dom e a dádiva entre parteiras do Amapá: uma abordagem etnográfica. Saúde e Sociedade, $28(1), 235-249$.

Cursino, T. P.; \& Benincasa, M. (2020). Parto domiciliar planejado no Brasil: uma revisão sistemática nacional. Ciência \& Saúde Coletiva, 25(4), $1433-1444$.

Davim, R. M. B., \& Menezes, R. M. P. (2001). Assistência ao parto normal no domicílio. Revista Latino-Americana de Enfermagem, 9(6), 62-68.

Denipote, A. G. M., Lacerda, M. R., Selleti, J. D. N., Tonin, L., \& Souza, S. R. R. K. (2020). Parto domiciliar planejado no Brasil: onde estamos e para onde vamos? Research, Society and Development, 9(8), e837986628.

Dias, M. A. B., \& Deslandes, S. F. (2006). Expectativas sobre a assistência ao parto de mulheres usuárias de uma maternidade pública do Rio de Janeiro, Brasil: os desafios de uma política pública de humanização da assistência. Cadernos de Saúde Pública, 22(12), 2647-2655.

Fabrizzio, G. C., Schmalfuss, J. M., Silveira, L., Peiter, C. C., Santos, J. L. G., Erdmann, A. L. (2019). Práticas obstétricas de uma parteira: contribuições para a gestão do cuidado de enfermagem à parturiente. Revista de Enfermagem do Centro-Oeste Mineiro, 9, e2892.

Frank, T. C., \& Pelloso, S. M. (2013). A percepção dos profissionais sobre a assistência ao parto domiciliar planejado. Revista Gaúcha de Enfermagem, 34(1), 22-29.

Kappaun, A., \& Costa, M. M. M. (2020). A institucionalização do parto e suas contribuições na violência obstétrica. Revista Paradigma, 29(1), 71-86.

Kruno, R. B.; \& Bonilha, A. L. L. (2004). Parto no domicílio na voz das mulheres: uma perspectiva à luz da humanização. Revista Gaúcha de Enfermagem, 25(3), 396-407.

Lessa, H. F., Tyrrell, M. A. R., Alves, V. H., Rodrigues, D. P. (2018). A opção pelo parto domiciliar planejado: uma opção natural desmedicalizada. Revista Online de Pesquisa Cuidado é Fundamental, 10(4), 1118-1122.

Lima, C. M., Pavoski, J., Silvestre, G. C. S. B., Nascimento, G. N. X., Magalhães, D. S. S., Ferro, R. B. C. (2021). Modelo de assistência ao parto normal: atuação das parteiras no Brasil. Enfermagem Brasil, (20)1, 109-123.

Limeira, J. B. R., Souza, G. C., Souza, M. B., Vieira, A. S., Alexandre, A. C. S., \& Leite-Salgueiro, C. D. B. (2018). A importância da humanização do parto realizada pelos enfermeiros obstetras para as parturientes: revisão integrativa. Revista Multidisciplinar e de Psicologia, 12(42), 308-321.

Marconi, M. A., \& Lakatos, E. M. (2017). Metodologia do trabalho científico. (8a ed.), Atlas.

Mattos, D. V., Vandenberghe, L., Martins, C. A. (2016). The obstetric nurse in a planned household birth. Revista de Enfermagem da UFPE, 10(2), 568-575.

Medeiros, M. L., Santos, T. M., Oliveira, M. P. L. C., Costa, A. W. S., Oliveira, E. N., Silva, M. A. R., \& Oliveira, R. F. (2020). O resgate da cultura dos partos domiciliares: uma revisão integrativa de literatura. Research, Society and Development, 9(4), e40942792.

Mocheuti, K. N., Zamboni, S. D., Silva, R. A., Rocha, R. P. S., Silvestre, G. C. S. B., \& Borges, A. P. (2020). Os significados atribuídos pela mulher ao trabalho das enfermeiras obstetras no parto domiciliar planejado. Research, Society and Development, 9(10), e019108237.

Nascimento, N. M., Progianti, J. M., Novoa, R. I., Oliveira, T. R., \& Vargens, O. M. C. (2010). Tecnologias não invasivas de cuidado no parto realizadas por enfermeiras: a percepção de mulheres. Escola Anna Nery, 14(3), 456-461.

Oliveira, T. R., Barbosa, A. F., Alves, V. H., Rodrigues, D. P., Dulfe, P. A. M., \& Maciel, V. L. (2020). Assistência ao parto domiciliar planejado: trajetória profissional e especificidades do cuidado da enfermeira obstétrica. Texto \& Contexto Enfermagem, 29 , e20190182.

Organização Mundial da Saúde (2015). Declaração da OMS sobre taxas de cesáreas. OMS.

Possati, A. B., Prates, L. A. Cremonese, L., Scarton, J., Neumaier, J., Alves, C. N., et al. (2017). Humanização do parto: significados e percepções de enfermeiras. Escola Anna Nery, 21(4), 1-6.

Rattner, D. (2009). Humanização na atenção a nascimentos e partos: breve referencial teórico. Interface: Comunicação, Saúde, Educação, $13(1)$, 595-602.

Sanfelice, C. F. O., Abbud, F. S. F., Pregnolatto, O. S., Silva M. G., Shimo, A. K. K. (2014). Revista da Rede de Enfermagem do Nordeste, $15(2)$, 362-370. 
Research, Society and Development, v. 10, n. 5, e11410514598, 2021

(CC BY 4.0) | ISSN 2525-3409 | DOI: http://dx.doi.org/10.33448/rsd-v10i5.14598

Santos, F. S., Sousa, L. C., Siqueira, L. S., Fontoura, I. G., Dias, I. C. C. M., \& Santos Neto, M. (2020). Percepções de puérperas sobre a assistência ao parto normal humanizado. Revista Científica de Enfermagem, 10(32), 217-228.

Santos, L. C. (2019). Parto domiciliar planejado: uma revisão bibliográfica. Revista Artigos.Com, 3, e1201.

Silva, T. M. A., Góis, G. A. S., Filgueiras, T. F., \& Candeia, R. M. S. (2019). Significados e práticas da equipe de Enfermagem acerca do parto humanizado: uma revisão de literatura. Brazilian Journal of Surgery and Clinical Research, 26(1), 90-94.

Silva, T. P. R., Dumont-Pena, E., Moreira, A. D., Camargos, B. A., Meireles, M. Q., Souza, K. V. et al. (2020). Fatores associados ao parto normal e cesárea em maternidades públicas e privadas: estudo transversal. Revista Brasileira de Enfermagem, 73(4), e20180996. 